\title{
H-TArget model: Early technology assessment for ext generation sequencing in oncology.
}

\author{
Valesca Retel, Manuela A Joore, Bram Ramaekers, Michel M van den Heuvel, Michiel Simon Van Der \\ Heijden and Wim van Harten
}

Netherlands Cancer Institute, Amsterdam, Netherlands; Department of Clinical Epidemiology and Medical Technology Assessment, Maastricht University Medical Centre, Maastricht, Netherlands; Maastricht University Medical Center, Maastricht, Netherlands; The Netherlands Cancer Institute, Amsterdam, Netherlands

\begin{abstract}
6604

Background:

Next Generation Sequencing (NGS) promises to find mutations (targets) in individual cancer patients, to subsequently prescribe targeted therapy. Currently, NGS is in development, the effects on choice of therapy and prognosis are still unclear, and the costs for targeted therapies are high. To accelerate the reimbursement process of NGS and potential new targeted therapies, and have a NGS-panel available for patients in the earliest possible stage, early Technology Assessment (TA) is performed to inform policy making around NGS in the Netherlands. One of the aims of the TA was to conduct a cost-effectiveness analysis.
\end{abstract}

\section{Methods:}

We constructed a target-based decision model (H-TArget) to estimate the cost-effectiveness of NGS versus single- and no testing. Standard- and targeted therapies in first and second line for 9 targets (BRAF, KRAS, NRAS, EGFR, ERBB/HER, MET, ROS, ALK, RET) over 3 tumor types (melanoma, non-small-cell lung cancer (NSCLC), colorectal cancer (CRC)) were incorporated. A Dutch healthcare perspective and a 5-year time horizon were adopted. Outcomes were incremental cost-effectiveness ratios (ICER) expressed in $€$ /quality adjusted life year (QALY). The threshold for cost-effectiveness is $80 \mathrm{k}$ in the Netherlands, which means that the concerning technology is cost-effective if the ICER is below this threshold. Expected Value of Partial Perfect Information $(E V P(P) I)$ was calculated to quantify the value of further research into particular subsets of uncertain parameters.

\section{Results:}

The expected ICER was $€ 65 \mathrm{k} / \mathrm{QALY}$ for melanoma, $€ 188 \mathrm{k} / \mathrm{QALY}$ for NSCLC, and $€ 103 \mathrm{k} / \mathrm{QALY}$ for CRC. As a weighted average to the three populations, the overall ICER yielded $€ 160 \mathrm{k} / \mathrm{Q} A L Y$. The EVPI was $€ 25 \mathrm{M}$ for melanoma, the subsets of parameters to focus on in future research were: $€ 2 \mathrm{M}$ together for failures, prevalence, survival, and $€ 23 \mathrm{M}$ for costs.

\section{Conclusions:}

At the moment, using NGS is only cost-effective for melanoma. This is mostly due to the high costs of targeted therapies and the fact that the effects are still small. Based on our findings, industry should strive for a significant cost reduction of targeted therapies or achieve a spectacular improvement in effectiveness, which could improve the cost-effectiveness. 